Cahiers

Balkaniques
Cahiers balkaniques

36-37 | 2008

L'image de la période ottomane dans les littératures balkaniques

\title{
Les possibilités pour la réconciliation
}

Comment tuer Apostol? de Slobodan Micković

The possibilities of reconciliation

\section{Vlada Urošević}

\section{OpenEdition}

Journals

Édition électronique

URL : https://journals.openedition.org/ceb/1494

DOI : 10.4000/ceb.1494

ISSN : 2261-4184

Éditeur

INALCO

Édition imprimée

Date de publication : 1 décembre 2008

Pagination : 113-118

ISBN : 978-2-85831-173-6

ISSN : 0290-7402

\section{Référence électronique}

Vlada Urošević, «Les possibilités pour la réconciliation », Cahiers balkaniques [En ligne], 36-37 | 2008, mis en ligne le 15 mai 2012, consulté le 06 juillet 2021. URL : http://journals.openedition.org/ceb/1494 ; DOI : https://doi.org/10.4000/ceb.1494

Ce document a été généré automatiquement le 6 juillet 2021.

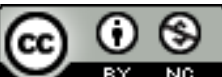

Cahiers balkaniques est mis à disposition selon les termes de la Licence Creative Commons Attribution - Pas d'Utilisation Commerciale 4.0 International. 


\title{
Les possibilités pour la réconciliation
}

\author{
Comment tuer Apostol? de Slobodan Micković \\ The possibilities of reconciliation
}

Vlada Urošević

1 Les cinq cents ans de domination ottomane sur la péninsule des Balkans n'ont évidemment pas été faciles pour les peuples de cette région, obligés de passer cette longue période dans une situation plus ou moins défavorable pour les droits collectifs et les droits de l'homme. Certes, cette période ouvrait les possibilités de l'enrichissement des cultures de ces peuples avec les éléments d'une autre civilisation qui apportait ses propres valeurs, mais, d'un autre côté, l'invasion venue d'Asie avait coupé les relations de ces peuples avec la civilisation de l'Europe de l'Ouest, en les séparant de leur milieu naturel. L'occupation ottomane avait de graves conséquences sur leur développement: sur les plans de la vie nationale, religieuse, culturelle. Les non-musulmans étaient, partout dans l'Empire, des citoyens de deuxième ordre, privés de nombreux droits, exposés à l'autocratie et aux brimades de toutes sortes. Et c'est pourquoi, dans la mémoire collective de tous les peuples balkaniques, cette période est restée comme un temps sombre, malheureux et néfaste.

Cette image est stéréotypée dans toutes les littératures balkaniques du XIX ${ }^{\mathrm{e}}$ siècle et de la première moitié du $\mathrm{XX}^{\mathrm{e}}$ siècle. Celles-ci contiennent des scènes d'humiliation, de mauvais traitements et, parfois, de grande cruauté de la part des oppresseurs envers les gens appartenant aux peuples oppressés. L'État ottoman est, dans cette mémoire, un véritable nid de corruption, caractérisé par l'absence de la légalité et le règne de l'anarchie. Le Turc, et surtout le Turc qui occupe une position de pouvoir, y est présenté comme un violent usurpateur, sans scrupule, corruptible et, assez souvent dans un esprit de vengeance - comme peu intelligent. La lutte pour la libération et le sentiment de triomphe dans les premières décennies de la liberté obtenue ont donné à ces attributs des caractères de lieux communs et, souvent, des traits assez grossiers. 
3 La littérature macédonienne a les mêmes caractéristiques. Le peuple macédonien est le dernier parmi les peuples balkaniques à être sorti de l'Empire ottoman. Et son rapport envers l'Empire est un peu différent. Dans les dernières années de la présence des Ottomans en Macédoine, après l'Huriet, il y eut même des chefs de la résistance contre le pouvoir ottoman, qui - craignant les revendications des autres États balkaniques sur le territoire de la Macédoine - soutinrent la position qu'il fallait rester dans l'Empire réformé, dans une sorte de fédération. Telle fut la position de Yané Sandanski (1872-1915) qui plaida pour la coopération avec les «Jeunes-Turcs » et qui entra même avec ses troupes armées dans Istanbul, en 1908, pour empêcher la tentative de contrerévolution du sultan. Sandanski cherchait des possibilités pour améliorer la position du peuple macédonien par des moyens politiques aussi devint-il président du Parti fédératif populaire qui avait ses députés au Parlement turc. Mais les guerres balkaniques mirent fin à ces projets.

4 La littérature macédonienne souffrait des mêmes clichés, concernant la présentation de la situation pendant la période ottomane, que les autres littératures balkaniques. Réduite parfois dans ses couleurs au noir et blanc, l'image du " joug turc », porteuse des simplifications et des idées communément admises, commence à changer vers les années $70 \mathrm{du} \mathrm{XXe}$ siècle. Très caractéristique à cet égard est un épisode du roman de Slavko Yanevski (1920-2000) Les Obstinés (1970), dont l'action se déroule en 1835. Dans cet épisode, on trouve une autre image d'un Turc : Seldjik-bey, venu d'Izmir, « un poète boiteux et appauvri qui notait et décrivait des coutumes et qui était un grand connaisseur des peuples et des langues balkaniques». Selon le portrait brossé par l'auteur, il se met du côté des paysans macédoniens révoltés. Il intervient en leur faveur auprès des autorités, menaçant les oppresseurs d'aller lui-même à Istanbul défendre la cause des oppressés. Et quand le pouvoir envoie des hommes armés pour l'amener chez le maire de la ville de Skopje, il tire sur eux de sa tour de pierre et périt après deux jours de combat inégal. Avec ce personnage du roman de Yanevski, on commence, dans la littérature macédonienne, à faire la différence entre un Turc et les représentants du pouvoir ottoman. De l'image «typisée » d'une nation, on passe aux personnages individualisés c'est un grand pas vers la présentation plus réelle et plus vivante de toute la période ottomane.

5 Dans le roman Le Livre du ciel (2000) de l'écrivain Krsté Tchatchanski (1949), l'image de cette période est très intensivement colorée. L'action est maintenant placée dans la deuxième moitié du XIX ${ }^{e}$ siècle, dans les années où on sent de plus en plus la décadence de l'Empire. Les caractéristiques de cette image sont l'autocratie des seigneurs féodaux, leur tendance à s'émanciper du pouvoir central, les intrigues et la rivalité entre eux et, même, des petites guerres qu'ils font pour se venger l'un de l'autre. Un haut fonctionnaire venu à Istanbul dit dans le roman, en pensant aux autorités locales : «Ils jouent à la guerre toujours quand ils veulent. Ils rongent le pays du dedans, ils font que l'Empire s'affaiblit... ». Évidemment les institutions de l'État ne fonctionnent pas. Il y a partout des attaques contre des caravanes et contre des voyageurs solitaires. Les pillages, les rapts, les meurtres sont des phénomènes quotidiens. Et le résultat est l'insécurité de tous - des musulmans comme des chrétiens.

6 Bien sûr, l'image de la situation générale dans les régions dont on parle est toujours la même - L'Empire est un organisme qui ne réussit pas à s'adapter à l'ambiance du monde nouveau et continue de répéter les mêmes tours dans les ornières du passé. « À travers cet espace entouré de sommets enneigés ne passent que des brigands et des loups, et nul 
droit n'est valable. Chacun est un pacha avec un toug, un loup aux crocs acérés ». "La troupe du bachibozouk entrait dans les villages, faisait des razzias dans les maisons, se livrant au pillage et au viol, enlevant même les plus belles filles et les jeunes mariées. Ils étaient sans merci ».

7 Il y a cependant des exceptions dans le cadre de cette image : on voit, comme dans le roman de Yanevski, des individus qui ne s'ajustent pas dans la mosaïque connue. Dans le roman de Tchachanski c'est Adnan-bey, rêveur et mélancolique, le gouverneur d'une petite région, au sud-ouest de la Macédoine. Le bonheur ne dépend pas pour lui de ses biens matériels et il est incompréhensible pour son entourage qui le trouve extravagant et farfelu. Il rêve de bâtir son sérail sur le Drim, sur le modèle de Chambord qu'il a vu sur une carte postale. Les autres hauts fonctionnaires du pouvoir ottoman ne l'aiment pas et disent de lui, sous le manteau, "qu'il est très proche des chrétiens, qu'il donne de l'argent pour les églises, qu'il fournit des armes à des non-croyants et qu'il se met lui-même à la tête des serdari pour protéger le peuple ». De ceux qui sont sous son autorité ses ennemis disent : «Ce sont des montagnards coriaces. Rebelles. Des chrétiens et des Turcs, aussi. Comme s'ils étaient un même peuple. Des Balkaniques! Ils sont soutenus par un mythe, par un conte, par le ciel! » Quand il entrera en conflit avec le pouvoir, il ripostera et périra. Son sérail sur la rivière sera détruit par des explosifs. Dans son rapport, le commandant des troupes qui ont combattu Adnan-bey écrira qu'Adnan-bey et Georges le Noir, le chef des rebelles chrétiens, sont un seul et même personnage. Et les chrétiens, de leur côté, diront, en cachette, que dans le sérail, avant qu'il ne soit détruit, on a trouvé - dans une chambre secrète - une icône du Christ avec un cierge qui brûlait devant. Évidemment, le romancier veut nous suggérer que le peuple macédonien savait faire la différence entre les oppresseurs qui tyrannisaient le peuple, et les Turcs qui se sentaient comme partie inséparable de la population du pays. Et vraiment, malgré des différences en plusieurs domaines, il y a dans le roman des exemples de représentants de ces deux populations vivant comme de bons voisins.

8 Le roman Comment tuer Apostol?, de l'écrivain macédonien Slobodan Micković (1935-2002), est paru en 1994. Habitué à l'image stéréotypée de l'Empire ottoman comme domaine où règnent le despotisme asiatique, l'obscurantisme et la corruption, le lecteur trouvera ici une nouvelle situation : à côté des représentants de l'ancienne Turquie, il y a maintenant, aussi, des "Jeunes-Turcs» - des gens qui pensent d'une façon différente et qui s'agitent en faveur d'une Turquie moderne et européanisée. Parmi ces derniers est Ouvaïs-bey, colonel dans l'armée turque et héros principal du roman. L'action du roman se déroule en 1908 - dans une période où, sous la pression des nouvelles forces dans la société turque, le sultan est obligé de permettre des réformes. Mais, parallèlement aux idées progressistes, en Ouvaïs-bey vivent des obsessions du passé : pendant son enfance, quelqu'un a tué son père et, selon les ouïdire, il s'agirait d'Apostol, chef d'un groupe de rebelles macédoniens qui se battent contre le pouvoir ottoman. Élevé dans la pensée de la vengeance, Ouvaïs-bey mènera plusieurs fois des actions pour s'emparer du chef légendaire des « comitadji ». Il finit par réussir à l'attraper. Mais, pendant l'agonie du rebelle blessé, Ouvaïs-bey apprend que ce n'est pas Apostol qui a tué son père - mais des brigands turcs. Toute la haine contre cet homme, tous ses projets pour se venger de lui - tout cela perd sa raison d'être. Il comprend qu'il existait une chance de réconciliation entre eux - mais que cette chance est manquée. Tout cela est un fatal malentendu. À ce même moment, le chef des rebelles sort une bombe cachée - et son explosion les tue tous les deux. L'idée de l'auteur est claire: les deux peuples, l'un oppresseur du moment, l'autre oppressé, pourraient demain vivre ensemble, égaux en droits, dans un pays réformé - mais cette 
prise de conscience est venue trop tard. Et on peut en tirer la morale : la formule de la multiethnicité implique l'oubli des anciennes rancunes et la révision des préjugés hérités du passé.

9 En conclusion, on peut dire que l'image de l'Empire ottoman dans la littérature macédonienne commence à se démarquer de la représentation habituelle dans les dernières décennies du $\mathrm{XX}^{\mathrm{e}}$ siècle. L'Empire reste, en principe, une geôle assez sombre pour le peuple macédonien, mais on commence à entrevoir - comme une lueur lointaine - des possibilités de réconciliation avec les Turcs, en qui on voit maintenant non seulement des occupants, mais, aussi, des individus qui peuvent avoir des qualités humaines et dont les valeurs doivent être respectées et estimées. En tout cas, les nombreux exemples - dans ces romans, mais aussi dans plusieurs autres - parlent de développement de la conscience relative à la nécessité de la vie commune sur un même sol.

\section{RÉSUMÉS}

Le roman Comment tuer Apostol ? de l'écrivain macédonien Slobodan Micković (1935-2002), paru en 1994, s'intéresse à l'image du « Jeune Turc ». Habitué à l'image stéréotypée de l'Empire ottoman comme période historique où règnent le despotisme asiatique, l'obscurantisme et la corruption, le lecteur y trouvera une nouvelle situation : à côté des représentants de l'ancienne Turquie, il y a maintenant, aussi, des "Jeunes Turcs» - des gens qui pensent d'une façon différente et qui luttent en faveur d'une Turquie moderne et européanisée. Parmi ces derniers est Ouvaïs-bey, colonel de l'armée turque, héros principal du roman. L'action du roman se déroule en 1908 - dans une période où, sous la pression des nouvelles forces dans la société turque, le sultan est obligé d'accepter des réformes. Mais, parallèlement aux idées progressistes, en Ouvaïs-bey vivent des obsessions du passé : durant son enfance, quelqu'un a tué son père, et, selon ouï-dire, ce serait Apostol, le voïvode d'un groupe de rebelles macédoniens qui se battent contre le pouvoir ottoman. Élevé avec la pensée de la vengeance, Ouvaïs-bey mènera plusieurs fois des actions pour saisir le chef légendaire des « comitadjis ». Il finit par l'attraper. Mais, pendant l'agonie du rebelle blessé, Ouvaïs-bey apprend qu'Apostol est innocent - les meurtriers sont des brigands turcs. Toute la haine qu'il a gardée contre cet homme, tous ses projets pour se venger - tout cela perd sa raison d'être. Il comprend qu'il existait une chance pour une possible réconciliation entre eux - mais que cette chance est manquée. Tout cela était un fatal malentendu. Au même moment, le chef des rebelles sort une bombe cachée. Tous deux meurent dans l'explosion. L'idée de l'auteur est claire: les deux peuples, l'un oppresseur et l'autre oppressé auraient pu vivre ensemble, égaux en droits, dans un pays réformé - mais cette prise de conscience est venue trop tard. La morale qui se dégage de ce roman est : la multiethnicité implique l'oubli des anciennes rancunes.

The novel comment tuer apostol (how to kill apostol) by the macedonian writer slobodan micković (1935-2002) first appeared in 1994. Anyone familiar with the cliché image of the ottoman empire as dominated by asian despotism, obscurantism and corruption will be surprised: alongside timeworn veterans of the sublime porte, there are the so-called young turks, innovators who fought to modernize and, if you will, europeanize their country. The young turks included ouvaïs-bey, an army colonel, the novel's protagonist. The story is set in 1908 when, under the pressure of 
new forces within turkish society, the sultan was forced to accept reform. In keeping with the ideas that were infiltrating what we now call turkey, ouvaïs-bey is haunted by his past: he was still a child when his father was murdered. The culprit was supposedly apostol, who belonged to a group of rebel macedonians who resisted the power of the ottomans. Raised with a taste for vengeance, ouvaïs-bey led numerous campaigns to capture the legendary chief of the 'comitadji'. He finally managed to arrest him, in order to find, just as the wounded rebel is dying in agony, that apostol is innocent and that his father's killers were actually turkish bandits. Thus all the hatred toward this man that he harboured, all his dreams of revenge, suddenly lose their meaning. Whether or not there'd been any chance of reconciliation, he lost it once and for all, he now realized. It was all a terrible misunderstanding. And then the rebel chief reveals a bomb and they all die in the explosion. The author's idea is thus clarified: the two peoples - the oppressors and the oppressed - could have peacefully coexisted, with equal rights, in a reformed society. But dawn broke too late. The moral of the story? Multiethnicity is all about burying hatchets.

\section{INDEX}

Index géographique : Macédoine, Skopje

Mots-clés : Bachi-bouzouk, Ottomans dans la littérature, littérature macédonienne, Comitadji, Majski Nikola Kirov (1880-1962), Micković Slobodan (1935-), Micković Slobodan (1935-2002), Jeunes-Turcs, Sandanski Yané (1872-1915), Yanevski Slavko (1920-2000), Tchatchanski Krsté, Tchatchanski Krsté (1949-2003), Yanevski Slavko (1920-2000)

Keywords : macedonian literature, Ottoman empire, Literature, Macedonia, Micković Slobodan (1935-), Skopje, Yanevski Slavko (1920-2000), Tchatchanski Krsté, Young Turk

Thèmes : Littérature

Index chronologique : Empire ottoman, vingtième siècle

motsclesmk МАКЕДОНИЈА, МЛАДОТУРСКАТА, ОТОМАНСКАТА ИМПЕРИЈА, СКОПЈЕ

motsclestr Makedonya, Üsküb, Genç Türkler, Osmanlı İmparatorluğu

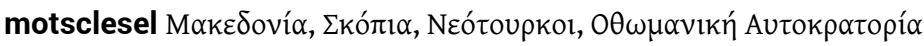

